\title{
Ergebnisse der Tieftemperaturforschung ${ }^{1}$

\author{
VII. Die Molwärmen der Alkalihalogenide $\mathrm{LiF}, \mathrm{NaCl}, \mathrm{KCl}, \mathrm{KBr}, \mathrm{KJ}, \mathrm{RbBr}$ und $\mathrm{RbJ}$ \\ von $10^{\circ}$ bis $273^{\circ}$ abs. $^{2}$
}

\author{
Von Klaus Clusius, ${ }^{3}$ Jochen Goldmann und Albert Perlick
}

(Z. Naturforschg. 4 a, 424-432 [1949]; eingegangen am 27. Januar 1949)

\begin{abstract}
Um den Gültigkeitsbereich der Debyeschen Kontinuumstheorie zu prüfen, wird die Molwärme der genannten Alkalihalogenide im Vakuumkalorimeter gemessen. Dazu dienen aus dem Schmelzfluß gezogene Kristallstäbe. Für keines der Salze ergibt sich eine konstante charakteristische Temperatur $\Theta_{\mathrm{D}}$. In einem $\Theta_{\mathrm{D}}$-T-Diagramm laufen die $\Theta_{D}$-Werte nach der $\Theta_{D}$-Achse hin bei tiefen Temperaturen fächerförmig auseinander. Das bedeutet mit sinkender Temperatur einen Anstieg der $\Theta_{\mathrm{D}}$-Werte bei hohen charakteristischen Temperaturen, bzw. einen Abfall bei niedrigen charakteristischen Temperaturen. Keines der drei Salze $\mathrm{LiF}, \mathrm{NaCl}$ und $\mathrm{KCl}$, die bis zu genügend kleinen $T / \Theta_{\mathrm{D}^{-}}$ Werten gemessen werden konnten, befolgt das $T^{3}$-Gesetz, vielmehr laufen die $\Theta_{\mathcal{D}}$-Werte durch ein Minimum, das dem Pseudo- $T^{3}$-Gebiet von Blackman entspricht. Bei allen Salzen tritt ein Maximum der $\Theta_{\mathrm{D}}$-Werte auf, das um so ausgeprägter erscheint, je niedriger die $\Theta_{\mathrm{D}}$-Werte sind. Die Bornsche Gittertheorie, in der von Blackman, Kellermann u. a. weitergeführten Form, ermöglicht eine quantitative Berechnung der spezif. Wärme beim $\mathrm{NaCl}$ und KCl. Eine Prüfung der übrigen Salze steht noch aus. Die praktische Brauchbarkeit der Debyeschen Funktion zur Extrapolation thermodynamischer Größen bis zum absoluten Nullpunkt wird durch die Ergebnisse der vorliegenden Arbeit nicht berührt, wenn man die bei $10^{\circ}$ abs geltenden $\Theta_{D}$-Werte zugrunde legt.
\end{abstract}

$\mathrm{D}$ ie klassische Quantentheorie für den Zusammenhang der Eigenfrequenzen von Gasen und festen Körpern mit dem Verlauf ihrer spezif. Wärme darf in den Grundzügen als abgeschlossen gelten. Damit hat die Anziehungskraft dieses einst im Brennpunkt des Interesses stehenden Gebiets stark abgenommen. Übriggeblieben ist im Augenblick nur die Diskussion über die Rolle der ,inneren“ Freiheitsgrade, die bei der gehemmten Rotation von Atomgruppen in der Molekel und im Gitter auftreten. Zu Unrecht, denn diese Aufgaben der Molekulardynamik sind keineswegs die einzigen, die in Verbindung mit der spezif. Wärme Aufmerksamkeit verdienen. Schon das Gitter von so einfach gebauten Körpern, wie es die Alkalihalogenide sind, zeigt Eigentümlichkeiten, die von dem klassischen Debyeschen Ansatz nicht quantitativ erfaßt werden.

Gewöhnlich unterschätzt màn die Schwierigkeiten und Einschränkungen, die einer umfassenden Prüfung der Debyeschen Theorie an einatomigem Versuchsmaterial in einem weiten Temperaturbereich entgegenstehen. Metalle sind bei

1 K. Clusius u. F. Konnertz, Ergebnisse der Tieftemperaturforschung. VI. Kalorimetrische Messungen der Verdampfungswärme des Sauerstoffs, Äthylens und Propylens. Z. Naturforschg. 4a, 117 [1949]. den tiefsten Temperaturen wenig geeignet, da sich hier der von Sommerfeld entdeckte Anteil der Elektronenwärme stark bemerkbar macht; die Atomwärme sinkt dann langsamer ab, als es das $T^{3}$-Gesetz verlangt ${ }^{4}$. Zudem wird bei manchen Metallen durch den Eintritt der Supraleitung das kalorische Verhalten besonders verwickelt. Von anderen Elementen wären an sich die einatomigen, regulär kristallisierenden Edelgase gute Versuchsobjekte. Dann müssen aber die Messungen wegen der niedrigen charakteristischen Temperaturen bis zu Heliumtemperaturen ausgedehnt werden. Außerdem fällt bei ihnen die Umrechnung von der Atomwärme konstanten Druckes, $C_{p}$, auf die konstanten Volumens, $C_{v}$, erheblich ins Gewicht; sie ist aber mangels geeigneter Unterlagen recht unsicher.

Beide Nachteile zeigen die Alkalihalogenide nicht. Nur muß man als eine andere Komplikation in Kauf nehmen, daß die Gitterpunkte abwech-

2 Diese Arbeit ist Hrn. Prof. R. W. P o h l, Göttingen, zum 60. Geburtstag gewidmet worden; sie war am 20. Juni 1944 bei der Z. physik. Chemie zur Veröffentlichung eingegangen, konnte dort aber nicht mehr erscheinen.

3 Zürich, Rämistr. 76.

4 S. dazu K. Clusius u. L. Sch a chinger, Z. Naturforschg. 2 a, 90 [1947]. 
selnd mit Bausteinen verschiedener Masse besetzt sind. Nach der Debyeschen Theorie ist dies nicht allzu schwerwiegend, denn mit sinkender Temperatur sollte der Einfluß der atomistischen Struktur wegen der zunehmenden Wellenlänge der Gitterschwingungen ohnehin immer mehr zurücktreten.

Derartige Untersuchungen sind schon von Nernst ${ }^{5}$ in der klassischen Zeit der Atomwärmeforschung an Natrium- und Kaliumchlorid ausgeführt worden. Die Ergebnisse zeigten zwischen den Temperaturen des flüssigen Wasserstoffs und der flüssigen Luft die praktische Brauchbarkeit der Debyeschen Theórie. Um jedoch zu einer genaueren Beurteilung der Sachlage zu kommen, mußte der Umfang des Beobachtungsmaterials erweitert werden. Wir untersuchten daher die Molwärme der Salze $\mathrm{LiF}, \mathrm{NaCl}, \mathrm{KCl}$, $\mathrm{KBr}, \mathrm{KJ}, \mathrm{RbBr}$ und $\mathrm{RbJ}$ in dem Temperaturbereich von $10^{\circ}$ abs. bis $273^{\circ}$ abs.

\section{Experimentelles}

1. Versuchsmaterial. Bei dem verwendeten Material handelte es sich um klare, durchsichtige und sprungfreie Stäbe, die aus Schmelzflußkristallen herausgespalten waren, ein Teil wurde noch auf der Drehbank zylindrisch bearbeitet. Die Kristalle waren aus den reinsten Salzen der Firmen Kahlbaum oder Merck gezüchtet.*

Die Molwärme wurde nach der Methode des Vakuumkalorimeters in der früher beschriebenen Anordnung ${ }^{6}$ gemessen, wobei die Stäbe selbst als Kalorimeter dienten. Dazu wurden sie mit einem seideisolierten Heizdraht von $0,03 \mathrm{~mm}$ Dicke aus Konstantan und mit einem $0,08 \mathrm{~mm}$ dicken Bleidraht zur Temperaturbestimmung bewickelt. Die Temperaturen von $10^{\circ}$ bis $80^{\circ}$ abs. wurden nach einer früheren Arbeit ${ }^{7}$, oberhalb von $80^{\circ}$ abs. nach den Tabellen der P.T.R. im Landolt-Börnstein, unter Heranziehung der Nernstschen $\alpha$-Regel berechnet. Der Eispunkt wurde zu $273,16^{\circ}$ abs. angenommen. Den Wärmekontakt mit der Kristalloberfläche vermittelte hauchdünn aufgetragener, farbloser Japanlack. Die fertigen Versuchskörper mußten vor der Verwendung mehrere Stunden bei $130^{\circ} \mathrm{C}$ trocknen, wodurch gleichzeitig der $\mathrm{Pb}$ Draht gealtert wurde. Das Gewicht des Konstantan-

\footnotetext{
* Sämtliche Kristalle, außer den Rubidiumverbindungen, wurden uns freundlichst von Hrn. Prof. R. W. P o h l, Göttingen, mit bekannter Freigebigkeit überlassen, wofür wir vielmals danken. Der Staßfurter Kaligesellschaft verdanken wir die leihweise Überlassung von Rubidiumjodid und Rubidiumbromid, die freilich nur in geringer Menge vorhanden waren und gerade zur Anfertigung kleinerer, verschiedentlich mit Sprüngen durchzogener Stäbe ausreichten. Ihre Herstellung hatte freundlicherweise Hr. Prof. v. Hip pel übernommen, wofür wir auch hier vielmals danken.
}

und Bleidrahtes sowie des Lacks war bekannt und wurde bei der Berechnung der Wärmekapazität berücksichtigt.

2. Besondere Aufmerksamkeit erforderte die „L a c kkorrektur" aus' einem anderen Grund. Der bei $130^{\circ} \mathrm{C}$ vorgenommene Trocknungsprozeß ist ein Vorgang, bei dem neben der Oxydation noch Verdunstungs- und Polymerisationserscheinungen eine Rolle spielen. Wir haben festgestellt, daß, je nach der Dauer und Geschwindigkeit der Trocknung, die Wärmekapazität ein und desselben Lacks bei den tiefsten Temperaturen (10-20 $0^{\circ}$ abs.) bis um $100 \%$ verschieden ausfallen kann, während der Unterschied bei $80^{\circ}$ abs. nur noch wenige Prozente beträgt. Es' war also unbedingt nötig, die Stäbe ganz gleichartig zu behandeln und die Lackkorrektur an einer Probe zu ermitteln, die auf ein Kupferrohr bekannter Wärmekapazität aufgetragen war. Die Versuchskörper wurden 2 Tage an der Luft getrocknet, dann 6 Stdn. im Trockenschrank auf $60^{\circ} \mathrm{C}$ und weitere 4 Stdn. auf $130^{\circ} \mathrm{C}$ erhitzt.

Eine Vorstellung von der Größe der Lackkorrektur gibt nachfolgender Auszug der spezif. Wärme des Lacks. Die Korrektur machte rund 5-mal kleinere Beträge aus', da der Lackanteil gewöhnlich nur $0,2 \mathrm{~g}$ betrug.

\begin{tabular}{cccccccc}
\hline$T^{0}$ & 12 & 20 & 30 & 40 & 50 & 60 & 70 \\
$c_{p}$ & 0,009 & 0,019 & 0,037 & 0,059 & 0,079 & 0,091 & 0,099
\end{tabular}

Tab. 1. Spezif. Wärme von Japanlaek.

3. Als Proben wurden benutzt:

KJ: Quaderförmiges Stück $12 \times 16 \times 49 \mathrm{~mm} ; 28,252 \mathrm{~g}$ $=0,1702$ Mol.

$\mathrm{KBr}$ : Zylindrischer Stab von $18 \mathrm{~mm} \varnothing$ und $47 \mathrm{~mm}$ Länge; $32,315 \mathrm{~g}=0,2715 \mathrm{Mol}$.

$\mathrm{KCl}$ : Zylindrischer Stab von $20,5 \mathrm{~mm} \varnothing$ und $55 \mathrm{~mm}$ Länge; $35,200 \mathrm{~g}=0,4721 \mathrm{Mol}$.

$\mathrm{NaCl}:$ Runder Stab von 18,5 mm $\varnothing$ und 57,5 mm Länge; Gewicht $31,143 \mathrm{~g}=0,5328$ Mol.

LiF: Quaderförmiges, etwas unregelmäßiges Stïck $31 \times 14 \times 15 \mathrm{~mm}$; Gewicht 16,038 $\mathrm{g}=0,6183 \mathrm{Mol}$.

Alle diese Stäbe waren rißfrei; dagegen zeigten folgende Versuchskörper Sprünge:

RbJ: Zylindrischer Stab von $19 \mathrm{~mm} \varnothing$ etwa $34,5 \mathrm{~mm}$ Länge; $34,705 \mathrm{~g}=0,3268 \mathrm{Mol}$.

RbBr: Zylindrischer Stab, unregelmäßiger Querschnitt, 20,530 g = 0,2483 Mol.

4. Der Temperaturausgleich war infolge der großen Wärmeleitfähigkeit der Kristallstäbe auch bei hohen Temperaturen gut; selbst bei den mit Sprüngen durchsetzten Rubidiumsalzen konnte erst oberhalb von

5 W. Nernst u. F. A. Lindemann, S.-B. preuß. Akad. Wiss., physik-math. Kl. 1911, 494; 1921, 1160; Z. Elektrochem. angew. physik. Chem. 17, 817 [1911].

${ }^{\circ}$ K. Clusius u. J. Goldmann, Z. physik. Chem., Abt. B 31, 256 [1936].

7 K. Cl u si us, Z. physik. Chem., Abt. B 4, 41 [1929]. 


\begin{tabular}{|c|c|c|c|c|c|c|c|}
\hline abs. & $\widetilde{C}_{\boldsymbol{p}}$ & $\widetilde{C}_{v}$ & $\Theta_{\mathrm{D}}$ & $T^{0}$ abs. & $\bar{C}_{p}$ & $\widetilde{C}_{v}$ & $\Theta_{\mathrm{D}}$ \\
\hline $18,8_{0}$ & $0,007_{3}$ & - & 750 & 89,1 & 1,197 & 1,193 & 610 \\
\hline $19,0_{4}$ & $0,007_{7}$ & - & 745 & 93,2 & 1,319 & 1,314 & 611 \\
\hline $20,8_{0}$ & $0,010_{3}$ & - & 740 & 98,3 & 1,479 & 1,472 & 612 \\
\hline 21,4 & $0,011_{8}$ & - & 728 & 106,9 & 1,749 & 1,738 & 611 \\
\hline $22,0_{5}$ & $0,012_{8}$ & - & 731 & 112,1 & 1,908 & 1,894 & 613 \\
\hline $22,1_{5}$ & $0,013_{4}$ & - & 721 & 117,2 & 2,062 & 2,045 & 612 \\
\hline 24,6 & $0,018_{5}$ & - & 719 & 122,4 & 2,217 & 2,197 & 613 \\
\hline 24,8 & $0,018_{8}$ & - & 722 & 127,6 & 2,365 & 2,341 & 614 \\
\hline 27 & 0,0 & 一 & 710 & 9 & 2,512 & 34 & 614 \\
\hline 2 & 0, & - & 704 & 4 & 2,6 & 6 & 616 \\
\hline 29,3 & 0,03 & - & 697 & 43,8 & 4 & & 615 \\
\hline 29,9 & $0,036_{3}$ & - & 5 & 149,1 & 6 & 3 & 14 \\
\hline 33,1 & $0,052_{8}$ & - & 685 & 153,4 & 3,050 & 3,002 & 614 \\
\hline 35,9 & $0,070_{1}$ & - & 674 & 159,5 & 3,165 & 3,112 & 617 \\
\hline 38,5 & $0,090_{\bar{\tau}}$ & - & 664 & 164,5 & 3,275 & 3,216 & 617 \\
\hline 40,7 & $0,109_{8}$ & - & 658 & 169,6 & 3,3 & 3,316 & 620 \\
\hline 43,4 & 0,13 & - & 647 & 174,7 & 3,4 & 3, & 625 \\
\hline 45,5 & 0,16 & - & 642 & 1 & 3,5 & 3, & 620 \\
\hline 4 & 0 . & - & 634 & 1 & 3, & & 622 \\
\hline 5 & 0 & - & 630 & & & & 618 \\
\hline 5 & 0 & - & 622 & 3 & 3,8 & & 625 \\
\hline 5 & 0 & - & 623 & 6 & 4 & & 628 \\
\hline 60 & 0 & - & 637 & & 9 & & 626 \\
\hline 62,7 & 0 & - & 613 & 7 & 9 & 99 & 627 \\
\hline 63,3 & 0,572 & - & 610 & 9 & 4,0 & 3,961 & 631 \\
\hline 66,4 & 0,566 & - & 613 & 216,3 & 4,1 & 4,027 & 632 \\
\hline 66,5 & 0,572 & 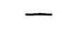 & 610 & 221,7 & 4,2 & 4,098 & 632 \\
\hline 69,6 & 0,64 & - & 611 & 226,9 & 4,269 & 4,130 & 639 \\
\hline 70,4 & 0,67 & - & 609 & 232,0 & 4,3 & 4,208 & 634 \\
\hline 72,7 & 0,730 & 0,729 & 607 & 237,4 & 4,4 & 4,258 & 638 \\
\hline $\mathbf{7 4 , 3}$ & 0,777 & 775 & 608 & 9 & 4,477 & $4, ?$ & 639 \\
\hline 75,6 & 0,817 & 815 & 606 & 5 & 4,537 & 4,366 & 641 \\
\hline 79,0 & 0,906 & 904 & 608 & 254,0 & 4,586 & 4,407 & 645 \\
\hline 81,9 & 0,99 & 0 & 607 & 9 & 4,644 & 4,457 & 645 \\
\hline 85 , & 1,08 & 1, & 610 & 265,9 & 4,718 & 4,520 & 645 \\
\hline 87,8 & 1,155 & 1,151 & 612 & 271,7 & 4,768 & 4,561 & 646 \\
\hline
\end{tabular}

Tab. 2. Durchschnittliche Atomwärme von Lithiumfluorid.

$100^{\circ}$ abs. eine geringe Überhitzung der Oberfläche beobachtet werden. Die Möglichkeit, kompakte, aus der Schmelze gewonnene Kristalle messen zu können; war für die Versuchsgenauigkeit äußerst günstig, weil alle Fehler fortfielen, die sich bei einem Kristallpulver durch schlechten Wärmeübergang, Adsorptionswärme des Füllgases im Kalorimeter und Wasserspuren einschleichen.

5 . Reduktion von $C_{p}$ a u f $C_{v}$. Da der unmittelbare kalorimetrische Versuch die Molwärme bei konstantem Druck $C_{p}$ liefert, mußten die Versuchsergebnisse auf $C_{v}$ umgerechnet werden, um zu einer mit der Theorie vergleichbaren Größe zu gelangen. Dies geschah mit ausreichender Genauigkeit durch die Gleichung

$$
C_{p} / C_{v}=1+A C_{p} T
$$

wobei der Proportionalitätsfaktor bei einer Temperatur - meist bei $0^{\circ} \mathrm{C}$ - durch Auswertung der exakten Beziehung

$$
C_{p} / C_{i}=1+\frac{c^{2} v_{0}}{C_{v} \%} T
$$

\begin{tabular}{|c|c|c|c|c|c|c|c|}
\hline$T$ & $\widetilde{C}_{p}$ & $\widetilde{C}_{v}$ & $\Theta_{\mathrm{D}}$ & $T^{0}$ abs. & $\widetilde{C}_{p}$ & $\widetilde{C}_{v}$ & $\Theta_{D}$ \\
\hline $10,9_{0}$ & 0,02 & & 299 & 80,7 & $\delta$, & $0, \mathrm{C}$ & 280 \\
\hline & 0,0 & & 292 & 89 & & & 283 \\
\hline & 0,035 & - & 297 & 93,9 & 3,984 & 35 & 282 \\
\hline $13, \tilde{5}_{0}$ & 0,044 & - & 297 & 98,5 & 4,138 & 4,082 & 282 \\
\hline & 0,055 & - & 295 & 103 & 74 & 2 & 282 \\
\hline & 0,067 & 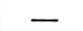 & 293 & 107 & & & 281 \\
\hline & $0,08 \mathrm{C}$ & - & 298 & 112 & & & 281 \\
\hline & 0,0 & & 289 & & & & 281 \\
\hline & 0,1 & & 288 & & & & 281 \\
\hline & 0 & & 290 & & & & 280 \\
\hline & 0 & & 89 & & & & 279 \\
\hline 21,2 & 0 & & 6 & & & & 278 \\
\hline 22,2 & 221 & & 84 & & & & 279 \\
\hline 25,1 &, 330 &, 329 & 80 & & & & 278 \\
\hline 27, & 42 & 4 & 76 & & 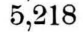 & & 278 \\
\hline 2 & 5 & 2 & 278 & & ; & & 278 \\
\hline 3 & 5 & 58 & 278 & & 2 & & 279 \\
\hline & c & 3 & 76 & & & & 279 \\
\hline & c & 7 & 76 & & & & 379 \\
\hline & & 8 & 75 & & & & 278 \\
\hline & & & 275 & & & & 8 \\
\hline & & & 275 & & & & 8 \\
\hline & & & 274 & & & & 282 \\
\hline & & & 275 & & & & 282 \\
\hline & & & 275 & & & & 283 \\
\hline 5 & & & 275 & & & & 85 \\
\hline 53 & 2 & & 5 & & & & 287 \\
\hline$\tilde{5}$ & 2 & 0 & 275 & & & & 28 \\
\hline 5 & 0,1 & 470 & 275 & & 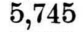 & & 29 \\
\hline & 96 & 61 & 277 & & & & 29 \\
\hline & 98 & & 277 & & & & 300 \\
\hline & 300 & & 277 & & & & 300 \\
\hline & & & 278 & & & & 303 \\
\hline & & & 279 & & & & 306 \\
\hline 80,2 & 502 & A & 280 & 267,5 & 5,864 & 5,561 & 291 \\
\hline
\end{tabular}

Tab. 3. Durchschnittliche Atomwärme von Natriumchlorid.

ermittelt wurde. Hierbei bedeuten $\alpha$ den kubischen Ausdehnungskoeffizienten und $x$ die kubische Kompressibilität.

\section{Diskussion der Ergebnisse}

1. Die Ergebnisse sind in den Tab. $2-8$ angeführt; neben der absoluten Temperatur und den durchschnittlichen Atomwärmen $C_{p}$ und $C_{v}$ ist noch die dazugehörige Debyesche charakteristische Temperatur $\Theta_{D}$ angegeben. Unter der durchschnittlichen Atomwärme ist bei den Alkalihalogeniden gerade die halbe Molwärme zu verstehen. Eine graphische Darstellung der Atomwärmen zeigt das bekannte Aussehen, so daß auf ihre Wiedergabe verzichtet werden kann. Es handelt sich durchweg um glatte Kurven; Umwandlungspunkte sind nicht vorhanden.

2. Von besonderem Interesse ist der Verlauf der $\Theta_{\mathrm{D}}$-Werte, der in Abb. 1 als Funktion der Temperatur $T$ wiedergegeben ist. Für den Fall der 


\begin{tabular}{|c|c|c|c|c|c|c|c|}
\hline$T^{0}$ abs. & $\widetilde{C}_{p}$ & $\widetilde{C}_{v}$ & $\theta_{\mathrm{D}}$ & $T^{0}$ abs. & $\widetilde{C}_{p}$ & $\widetilde{C}_{v}$ & $\Theta_{D}$ \\
\hline $10,8_{9}$ & $0,05_{5}$ & - & 222 & 105,8 & $4,78_{3}$ & $4,72_{8}$ & 234 \\
\hline $11,1_{6}$ & $0,0 \overline{5}_{9}$ & - & 222 & 110,7 & $4,89_{0}$ & $4,82_{9}$ & 232 \\
\hline $12,6_{1}$ & $0,08_{9}$ & - & 219 & 116,0 & $4,96_{0}$ & $4,89_{5}$ & 235 \\
\hline $12,7_{3}$ & $0,09_{1}$ & - & 219 & 121,1 & $5,09_{9}$ & $5,92_{7}$ & 228 \\
\hline $14,1_{8}$ & $0,12_{8}$ & - & 218 & 125,8 & $5,13_{9}$ & $5,06_{1}$ & 231 \\
\hline $14,2_{6}$ & $0,12_{8}$ & - & 219 & 130,7 & $5,22_{2}$ & $5,14_{0}$ & 228 \\
\hline $15,7_{1}$ & $0,18_{4}$ & - & 214 & 136,1 & $5,30_{8}^{2}$ & $5,22_{0}$ & 225 \\
\hline $15,7_{2}$ & $0,17_{2}$ & - & 219 & 142,3 & $5,34_{8}^{\circ}$ & $5,25_{6}$ & 228 \\
\hline $17,0_{8}$ & $0,21_{9}$ & - & 219 & 9 & $5,43_{9}$ & $5,33_{7}$ & 223 \\
\hline $18,2_{4}$ & $0,26_{5}$ & - & 219 & 155,3 & $5,45_{7}$ & $\mathbf{5}, 35_{0}$ & 230 \\
\hline $18,5_{2}$ & $0,27_{5}$ & - & 219 & 61,7 & $5,51_{3}$ & $5,39_{8}$ & 230 \\
\hline 19,98 & $0,34_{0}$ & - & 221 & 168,2 & $5,60_{1}$ & $5,48_{1}$ & 219 \\
\hline 22,7 & $0,49_{0}$ & 一 & 221 & 174,5 & $\mathbf{5}, \mathbf{5} 4_{0}$ & $5,41_{9}$ & 242 \\
\hline 24,8 & $0,62_{1}$ & - & 221 & 176,9 & $5,69_{4}$ & $5,56_{1}$ & 209 \\
\hline 28,2 & $0,94_{6}$ & - & 213 & 178,3 & $5,64_{6}$ & $5,51_{7}$ & 223 \\
\hline 31,5 & $1,11_{4}$ & $1,11_{3}$ & 223 & 184,5 & $5,67_{1}$ & $5,33_{7}$ & 225 \\
\hline 34,9 & $1,38_{8}$ & $1,38_{6}$ & 223 & 191,6 & $5,71_{0}$ & $5,56_{4}$ & 225 \\
\hline 41,2 & $1,89_{8}$ & $1,89_{5}$ & 224 & 198,4 & $5,74_{5}$ & $5,59_{8}$ & 222 \\
\hline 45,5 & $2,23_{3}$ & $2,22_{8}$ & 225 & 201,8 & $\mathbf{5}, 7 \check{5}_{0}$ & $5,59_{9}$ & 226 \\
\hline 50,1 & $2,56_{4}$ & $2,55_{7}$ & 226 & 204,3 & $5,77_{4}$ & $5,61_{6}$ & 223 \\
\hline 55,3 & $2,92_{9}$ & $2,91_{8}$ & 226 & 207,3 & $5,76_{8}$ & $5,61_{0}$ & 228 \\
\hline 61,3 & $3,27_{3}$ & $3,2 \check{5}_{9}$ & 227 & 213,4 & $5,86_{2}$ & $5,69_{8}$ & 202 \\
\hline 66,5 & $3,53_{1}$ & $3,51_{3}$ & 229 & 220,4 & $5,84_{7}$ & $5,67_{8}$ & 219 \\
\hline 71,8 & $3,60_{1}$ & $3,58_{0}$ & 242 & 227,1 & $5,8 \tilde{0}_{1}$ & $5,67_{7}$ & 224 \\
\hline 76,8 & $3,96_{7}$ & $3,93_{9}$ & 231 & 233,2 & $5,88_{1}$ & $5,70_{1}$ & 217 \\
\hline 81,0 & $4,12_{0}$ & $4,08_{8}$ & 229 & 239,6 & $5,91_{4}$ & $5,72_{2}$ & 216 \\
\hline 90,4 & $4,4 \tilde{5}_{6}$ & $4,41_{4}$ & 229 & 244,9 & $5,90_{7}$ & $5.71_{8}$ & 221 \\
\hline 94,2 & $4,49_{6}$ & $\mathbf{4}, \mathbf{4} \overline{\boldsymbol{n}}_{0}$ & 235 & 251,6 & $5,94_{9}$ & $5,75_{1}$ & 212 \\
\hline 98 & $4,62_{5}$ & $4,57_{8}$ & 232 & 259,2 & $5,97_{8}$ & $5,76_{6}$ & 208 \\
\hline 101,7 & $4,67_{0}$ & $4,61_{9}$ & 236 & 267,6 & $6,02_{9}$ & $5,81_{1}$ & 190 \\
\hline
\end{tabular}

Tab. 4. Durchschnittliche Atomwärme von Kaliumchlorid.

\begin{tabular}{|c|c|c|c|c|c|c|c|}
\hline$T^{0}$ abs. & $\widetilde{C}_{p}$ & $\bar{C}_{v}$ & $\Theta_{\mathrm{D}}$ & $T^{\circ}$ abs. & $\widetilde{C}_{p}$ & $\widetilde{C}_{v}$ & $\Theta_{D}$ \\
\hline $10,2_{7}$ & $0,32_{7}$ & - & 115 & 59,9 & $4,40_{7}$ & $4,36_{9}$ & 154 \\
\hline $10,6_{3}$ & $0,35_{7}$ & 一 & 115 & 66,8 & $4,63_{6}$ & $4, \bar{ก} 8_{\tau}$ & 157 \\
\hline $11,0_{9}$ & $0,38_{4}$ & - & 117 & 73,6 & $4,80_{0}$ & $4,74_{6}$ & 161 \\
\hline $11, \overline{5}_{5}$ & $0,44_{2}$ & - & 125 & 79,7 & $4,88_{6}$ & $4,82_{2}$ & 168 \\
\hline $12,0_{1}$ & $0,47_{9}$ & - & 118 & 86,0 & $4,9 \check{z}_{6}$ & $4,88_{\tilde{\tilde{j}}}$ & 177 \\
\hline $12,3_{5}$ & $0,52_{3}$ & - & 117 & 87,1 & $4,96_{2}$ & $4,89_{4}$ & 177 \\
\hline $12,9_{5}$ & $0,56_{5}$ & - & 119 & 92,6 & $5,06_{5}$ & $4,98_{8}$ & 178 \\
\hline $13,2_{1}$ & $0,62_{3}$ & - & 118 & 99,1 & $5,16_{\mathrm{a}}$ & $5,07_{9}$ & 180 \\
\hline $13,9_{2}$ & $0,68_{3}$ & - & 120 & 106,4 & $5,28_{5}$ & $5,18_{5}$ & 180 \\
\hline $14,1_{9}$ & $0,71_{6}$ & - & 120 & 113,8 & $5,36_{4}$ & $5,26_{0}$ & 182 \\
\hline $14,9_{9}$ & $0,78_{8}$ & - & 122 & 120,8 & $5,42_{6}$ & $5,31_{4}$ & 185 \\
\hline $15,1_{3}$ & $0,81_{5}$ & - & 121 & 12,75 & $5,46_{4}$ & $5,34_{2}$ & 190 \\
\hline $16,1_{4}$ & $0,90_{1}$ & - & 125 & 132,4 & $5,57_{3}$ & $\overline{5}, 43_{9}$ & 180 \\
\hline $16,5_{4}$ & $0,94_{1}$ & - & 125 & 139,2 & $5,58_{2}$ & $5,44_{2}$ & 181 \\
\hline $17,3_{1}$ & $1,01_{9}$ & - & 127 & 149,4 & $5,622_{9}$ & $5,47_{8}$ & 195 \\
\hline $17, \tau_{9}$ & $1,06_{3}$ & - & 128 & 159,9 & $\mathbf{5 , 6 5 _ { 5 }}$ & $5,49_{1}$ & 206 \\
\hline $18,7_{8}$ & $1,15_{7}$ & & 130 & 167,9 & $5,70_{8}$ & $5, \overline{3} 3_{5}$ & 205 \\
\hline $19,1_{7}$ & $1,20_{2}$ & & 131 & 176,2 & $5,73_{2}$ & $5,54_{5}$ & 213 \\
\hline 20,4 & $1,36_{5}$ & $1,36_{3}$ & 132 & 186,3 & $5,75_{2}$ & $5,55_{4}$ & 222 \\
\hline 22,4 & $1,57_{8}$ & $1,57_{6}$ & 135 & 198,6 & $\mathbf{5}, 79_{6}$ & $5,58_{0}$ & 229 \\
\hline 26,2 & $2,0 \mathbf{4}_{0}$ & $2,03_{8}$ & 137 & 219,1 & $5,87_{6}$ & $5,63_{6}$ & 231 \\
\hline 30,5 & $2,47_{4}$ & $2,47_{0}$ & 141 & 238,0 & $5,96_{4}$ & $5,70_{0}$ & 224 \\
\hline 35,0 & $2,90_{9}$ & $2,90_{0}$ & 156 & 248,7 & $6,14_{6}$ & $5,8 \check{\partial}_{2}$ & 150 \\
\hline 40,4 & $3,33_{8}$ & $3,32_{2}$ & 147 & 258,2 & $6,11_{0}$ & $5,80_{8}$ & 184 \\
\hline 46,2 & $3,72_{8}$ & $3,70_{7}$ & 150 & 269,9 & $6,23_{7}$ & $5,90_{4}$ & 108 \\
\hline 52,5 & $4,09_{0}$ & $4,06_{0}$ & 152 & & & & \\
\hline
\end{tabular}

Tab. 6. Durchschnittliche Atomwärme von Kaliumjodid.

\begin{tabular}{|c|c|c|c|c|c|c|c|}
\hline abs. & $\widetilde{C}_{p}$ & $\widetilde{C_{v}}$ & $\Theta_{\mathrm{D}}$ & $\boldsymbol{T}^{0}$ abs. & $\widetilde{C}_{p}$ & $\widetilde{C}_{v}$ & $\Theta_{\mathrm{D}}$ \\
\hline $3_{7}$ & $0,19_{6}$ & - & 52 & 7 & $4,51_{9}$ & $\mathbf{4}, \mathbf{4 0} 0$ & 177 \\
\hline & 0 & - & 153 & & & & 182 \\
\hline $12,1_{3}$ & $0,233_{1}$ & - & 153 & 84,3 & 4,8 & $4,77_{0}$ & 182 \\
\hline $12,4_{4}$ & $0,24_{0}$ & - & 153 & 84,7 & $4,85_{2}$ & $4,79_{9}^{\circ}$ & 180 \\
\hline $12,9_{1}$ & $0,27_{5}$ & - & 153 & 89,4 & $4,93_{5}$ & $4,87_{6}$ & 183 \\
\hline $13,0_{5}$ & $0,28_{5}$ & - & 153 & 94,4 & $5,05_{4}$ & $4,99_{0}$ & 181 \\
\hline $13,6_{5}$ & $0,32_{0}$ & - & 154 & 99,0 & $5,15_{0}$ & $5,08_{0}$ & 180 \\
\hline $14,0_{0}$ & $0,34_{3}$ & - & 154 & 103,7 & 5,2 & 5,1 & 178 \\
\hline 14,1 & $0,35_{2}$ & - & 154 & 108,8 & 5,3 & $5,23_{6}$ & 177 \\
\hline 14 & $0,40_{7}$ & - & 154 & 1 & $5,40_{0}$ & $5,31_{1}$ & 175 \\
\hline 15 & $0,42_{6}$ & - & 156 & 1 & 5, & 5,3 & 74 \\
\hline 15,4 & 0,4 & $\div$ & 156 & 1 & $\mathbf{5}, \mathbf{5} 3_{4}$ & 5,4 & 71 \\
\hline $16,3_{7}$ & 0,4 & 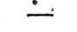 & 158 & 9 & $5,59_{0}$ & $5,48_{2}$ & 169 \\
\hline $16,5_{9}$ & $0,5 \mathbf{1}_{8}$ & - & 158 & 135,3 & $5,63_{8}$ & $5,52_{3}$ & 167 \\
\hline $16,7_{9}$ & $0,53_{9}$ & - & 157 & 140,6 & $5,67_{8}$ & $5,55_{7}$ & 167 \\
\hline $17,8_{9}$ & $0,60_{9}$ & - & 160 & 146,1 & $5,72_{4}$ & $5, \check{5} 9_{6}$ & 164 \\
\hline $18,0_{1}$ & $0,62_{6}$ & - & 158 & 155,9 & $5,79_{5}$ & $5,65_{2}$ & 160 \\
\hline $18,3_{5}$ & $0,64_{7}$ & - & 161 & 16. & $5,81_{3}$ & $5,66_{8}$ & 162 \\
\hline 19,5 & $0,74_{2}$ & - & 162 & 167,0 & $5,85_{7}$ & $5,70_{4}$ & 156 \\
\hline 19,6 & $0,75_{7}$ & - & 162 & 173,1 & $5,88_{2}$ & $5,72_{2}$ & 156 \\
\hline 21,7 & $0,96_{2}$ & - & 162 & 179,7 & $5,90_{0}$ & $5,73_{3}$ & 1.57 \\
\hline 2 & $1,2 \mathbf{4}_{9}$ & 1,2 & 166 & 4 & $5,94_{3}$ & $5,76_{8}$ & 50 \\
\hline 2 & $1,53_{1}$ & $1,52_{9}$ & 167 & 8 & $5,97_{3}$ & $5,79_{0}$ & 115 \\
\hline 2 & $1,77_{7}$ & $1,77_{4}$ & 169 & 198,5 & $5,98_{7}$ & 579 & 146 \\
\hline 361 & $2,38_{3}$ & $2,37_{8}$ & 173 & 205,1 & $6,00_{0}$ & $5,80_{3}$ & 148 \\
\hline 38.9 & $2,65_{4}$ & $2,64_{7}$ & 171 & 211,9 & $6,01_{7}$ & $\check{5}, 81_{3}$ & 148 \\
\hline 423 & $3,12_{5}$ & $3,11_{4}$ & 164 & 219,2 & $6,03_{6}$ & $5,82_{3}$ & 148 \\
\hline 4 & $3,18_{5}$ & 3,1 & 173 & 225,9 & $6,05_{6}$ & $5,83_{5}$ & 145 \\
\hline 49 & 3,42 & 34 & 174 & 232,8 & $6,06_{5}$ & $5,83_{7}$ & 148 \\
\hline 5 & 3,64 & 3,6 & 176 & 246,8 & $6,10_{7}$ & $5,86_{1}$ & 140 \\
\hline 56,2 & 3,8 & 3,8 & 17 ๖ & 254,0 & 6,11 & $5,86^{1}$ & 143 \\
\hline 59,8 & $4,04_{1}$ & $4,01_{5}$ & 175 & 262,2 & $6,1 b_{1}$ & $5,89_{5}$ & 120 \\
\hline 63,8 & $4,21_{7}$ & $4,18_{7}$ & 176 & 269,9 & $6,18_{3}$ & $5,90_{8}$ & 109 \\
\hline 67,8 & $4,37_{7}$ & $4,34_{2}$ & 176 & & & & \\
\hline
\end{tabular}

Tab. 5. Durchschnittliche Atomwärme von Kaliumbromid.

uneingeschränkten Gültigkeit der Debyeschen Theorie sollte der $\Theta_{D}$-Wert für ein bestimmtes Salz konstant und unabhängig von der Temperatur sein, d.h. die $\Theta$-Werte sind Parallelen zur Abszisse. Man erkennt nun, daß ein solcher einheitlicher $\Theta_{D}$-Wert in keinem Falle existiert, obwohl alle Salzé dem regulären System vom NaClTyp angehören, für das die Debyesche Funktion im allgemeinen recht gut gilt. Es läßt sich höchstens für jedes Alkalihalogenid ein ungefährer Bereich angeben, in dem der $\Theta_{D}$-Wert liegt, wobei aber für die Größe der Unstimmigkeit eine einfache Gesetzmäßigkeit nicht ohne weiteres zu erkennen ist. Ein eigenartiges Zusammentreffen hat es gefügt, daß die schon länger bekannten Molwärmen des $\mathrm{NaCl}$ und $\mathrm{KCl}$ in einem mittleren Temperaturbereich durch verhältnismäßig geringe Abweichungen von der Debyeschen Funktion ausgezeichnet sind. Beide Salze haben daher immer als Kronzeugen für die Brauchbarkeit der Kon- 


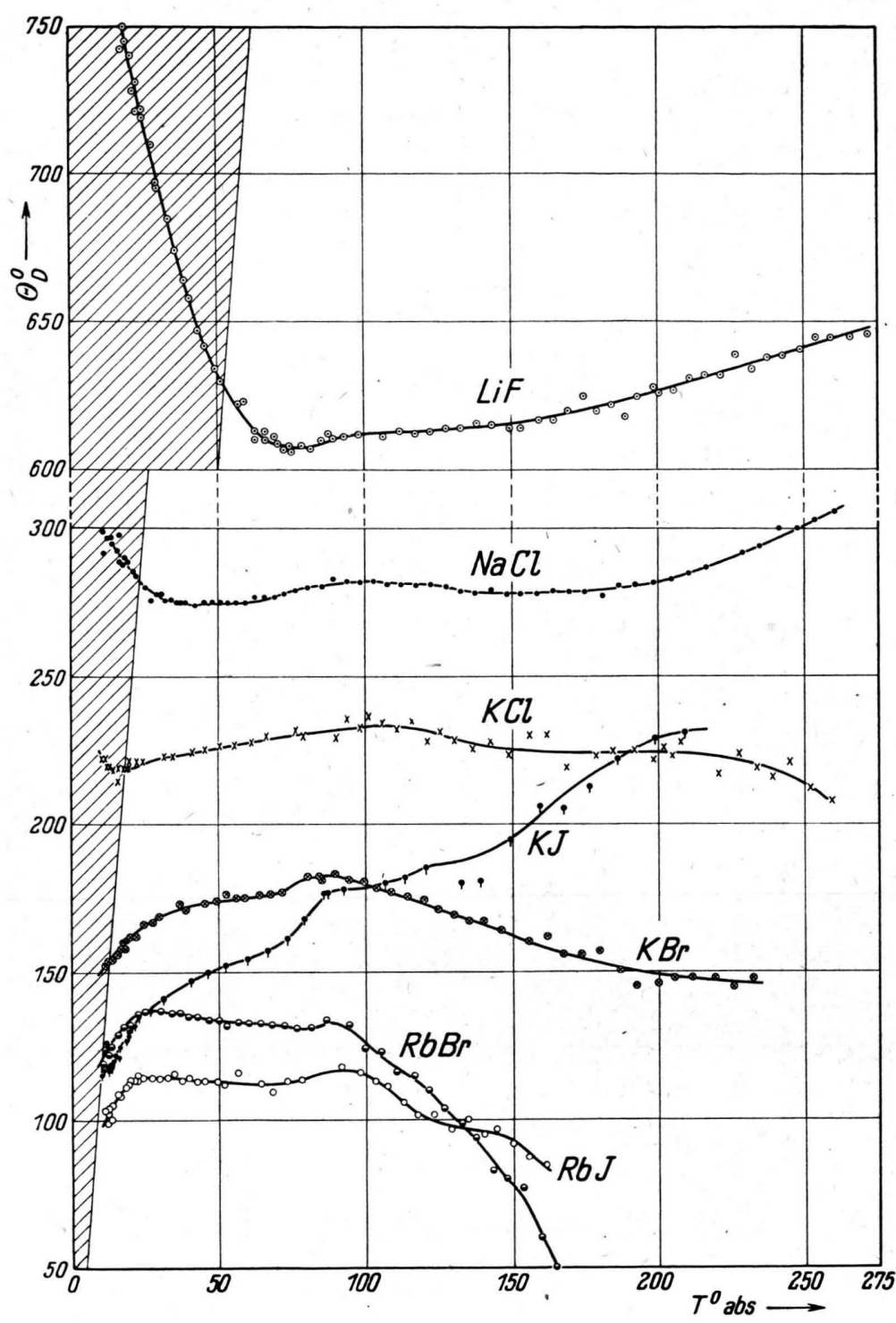

Abb. 1. Verlauf der Debyeschen charakteristischen Temperatur $\Theta_{D}$ bei den Alkalihalogeniden. Im schraffierten Bereich sollte das $T^{3}$-Gebiet mit konstantem $\Theta_{D}$-Wert erreicht sein, was bei $\mathrm{LiF}, \mathrm{NaCl}$ und $\mathrm{KCl}$ offensichtlich nicht zutrifft.

scheiden. Tatsächlich ist der Gang beim KJ mit dem Massenverhältnis $\mathrm{K}: \mathrm{J}$ $=1: 3,26$ besonders ausgeprägt. Ein ähnliches Verhalten wurde schon früher an den gleichfalls in NaCl-Struktur kristallisierenden Silbersalzen $\mathrm{AgCl}$ und in schwächerem Maße an $\mathrm{AgBr}$ beobachtet $^{8,9}$. Andererseits fällt auf, daß sich beim $\mathrm{RbBr}$ die $\Theta_{\mathrm{D}}$-Werte in der gleichen Richtung und Größenordnung ändern wie beim $\mathrm{RbJ}$, obwohl beim Bromid die Atommassen von Anion und Kation nahezu gleich groß sind $(\mathrm{Rb}: \mathrm{Br}$ $=1: 0,935)$, während sie sich beim Jodid um das 1,49-fache unterscheiden.

3. Die Beobachtungen der Molwärme liefern also ein Werteintervall für die Grenzfrequenzen $\Theta_{\mathrm{D}}$. Unter diesen Umständen muß ein Vergleich mit den aus elastischen Daten, aus dem Schmelzpunkt und den Reststrahl- bzw. Absorptionsfrequenzen berechneten $\Theta_{\mathrm{D}^{-}}$ Werten vorwiegend orientierenden Charakter haben. In Tab. 9 ist eine solche Gegenüberstellung vorgenommen. Die kalorischen $\Theta_{\mathrm{D}}$-Werte sind für das Intervall von $10^{\circ}$ abs. bis zu der Temperatur angegeben, bei der die $C_{v}$-Werte 5,5 cal erreichen. Eine Berücksichtigung höherer Temperaturen hat wenig Sinn, da dann die Voraussetzung rein harmonischer Schwingungen schlechter erfüllt ist, und kleine Meßfehler die $\Theta_{D^{-}}$ Werte stark beeinflussen.

Bei der Berechnung der charakteristischen Temperatur aus elastischen Daten übergehen wir die älteren Ansätze von Madelung, Sutherland, Einstein, Grüneisen u. a. Wir ergreifen vielmehr die Möglichkeit, den von Blackman ${ }^{10}$

tinuumstheorie gegolten; doch verlieren sie diese Eigenschaft bei einer schärferen Analyse und bei der Ausdehnung der Messungen auf tiefe Temperaturen.

Im allgemeinen darf man erwarten, daß der Gang in den $\Theta_{D}$-Werten um so größer ist, je mehr sich die Massen von Kation und Anion unter-

8 Für AgCl vgl. K. Clusius u. P. Harteck, Z. physik. Chem. 134, 260 [1928].

${ }^{9}$ Für AgBr vgl. A. Eucken, K. Clusius u. H. Wo it i n e ck, Z. anorg. allg. Chem. 203, 48 [1931]. für das kubische System abgeleiteten Ausdruck

$$
\Theta_{\mathrm{e}}=\frac{h}{2 \pi k} \sqrt{\frac{5 r_{0}}{\%}\left(\frac{1}{m_{\mathrm{A}}}+\frac{1}{m_{\mathrm{K}}}\right)}
$$

auf seine Brauchbarkeit hin zu prüfen. In ihm bedeuten $m_{\mathrm{A}}$ und $m_{\mathrm{K}}$ die Masse von Anion und Kation, $r_{0}$ ihren Abstand, $x$ die kubische Kompressibilität und $h$ die Plancksche, $k$ die Boltzmannsche Konstante. Diese Beziehung kann hier

${ }^{10}$ M. B l a c km a n, Proc. Roy. Soc. [London] 181, 58 [1942]. 


\begin{tabular}{|c|c|c|c|c|c|c|c|}
\hline$T^{0}$ abs. & $\widetilde{C}_{p}$ & $C_{v}$ & $\Theta_{\mathrm{D}}$ & $T^{0}$ abs. & $\bar{C}_{p}$ & $\widetilde{C}_{v}$ & $\Theta_{\mathrm{D}}$ \\
\hline $10,5_{2}$ & $0,31_{9}$ & - & 119 & 110,6 & $5,71_{7}$ & $5,63_{8}$ & 116 \\
\hline $11,6_{2}$ & $0,37_{3}$ & 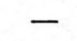 & 124 & 116,0 & $5,75_{9}$ & $5,67_{4}^{\circ}$ & 115 \\
\hline $12,4_{7}$ & $0,47_{1}$ & - & 122 & 121,4 & $\overline{5}, 81_{0}$ & $5,72_{0}$. & 110 \\
\hline $13,8_{7}$ & $0, \tilde{0} 9_{9}$ & - & 125 & 126,8 & $5,8 \overline{0}_{5}$ & $\mathbf{5}, \mathbf{7} \tilde{\mathbf{n}}_{9}$ & 104 \\
\hline $15,4_{6}$ & $0,73_{7}$ & - & 129 & 132,2 & $5,89_{5}$ & $5,79_{3}$ & 99 \\
\hline $17,4_{6}$ & $0,94_{2}$ & 一 & 132 & 137,6 & $5,92_{5}$ & $5,81_{9}$ & 94 \\
\hline $19,8_{6}$ & $1,22_{4}$ & - & 135 & 143,1 & $5,96_{1}$ & $\mathbf{5}, 84_{9}$ & 83. \\
\hline 2 & $1,52_{4}$ & $1,52_{3}$ & 136 & 4 & $5,98_{8}$ & $5,87_{1}$ & 80 \\
\hline 24,8 & $\cdot 1,87_{8}$ & $1,87_{6}$ & 136 & 8 & $6,00_{4}$ & $5,88_{2}$ & 77 \\
\hline 27,3 & $2,51_{0}$ & $2, \overline{5} 0_{6}$ & 130 & 159,3 & $6,04_{4}$ & $5,91_{6}$ & 60 \\
\hline 30,3 & $2,57_{0}$ & $2,56_{6}$ & 137 & 164,8 & $6,06_{2}$ & $5,92_{9}$ & 50 \\
\hline 33,2 & $2,90_{1}$ & $2,89_{5}$ & 136 & 170,5 & $6,07_{4}$ & $5,93_{6}$ & 44 \\
\hline 36,2 & $3,21_{6}$ & $3,20_{8}$ & 136 & 176,0 & $6,09_{0}$ & $5,94_{6}$ & 33 \\
\hline 39,4 & $3, \mathbf{5 3}_{4}$ & $3,52_{3}$ & 135 & 181,7 & $6,10_{2}$ & - & - \\
\hline 42,8 & $3,81_{7}$ & $3,80_{3}$ & 135 & 187,5 & $6,12_{5}^{-}$ & - & - \\
\hline 46,2 & $4,04_{9}$ & $4,03_{2}$ & 134 & 193,3 & $6,07_{1}$ & - & - \\
\hline 49,6 & $4,26_{5}$ & $4,24_{5}$ & 134 & 199,2 & $6,16_{5}$ & - & - \\
\hline 52,8 & $4,46_{3}$ & $\mathbf{4 , 4 4 _ { 3 }}$ & 132 & 200,1 & $6,17_{2}$ & - & - \\
\hline 56,7 & $4,61_{3}$ & $4,58_{7}$ & 133 & 206,1 & $6,19_{5}$ & - & - \\
\hline 60,5 & $4,77_{0}$ & $4,73_{0}$ & 133 & 2 & $6,19_{8}$ & 一 & - \\
\hline 64,5 & $4,89_{4}$ & $4,86_{0}$ & 133 & 217,9 & $6,21_{1}$ & - & - \\
\hline 68,5 & $5,01_{7}$ & $4,97_{9}$ & 132 & 223,7 & $6,21_{2}$ & 一 & - \\
\hline 72,7 & $5,11_{8}$ & $5,07_{6}$ & 132 & 229,6 & $6,22_{7}$ & 一 & - \\
\hline 76,9 & $5,20_{9}$ & $5,16_{3}$ & 131 & 235 & $6,24_{8}$ & - & - \\
\hline 81,5 & $5,30_{0}$ & $5,25_{0}$ & 131 & 242,4 & $6,25_{4}$ & - & - \\
\hline 86,5 & $\mathbf{5}, \mathbf{3 5 _ { 0 }}$ & $\hbar, 29_{5}$ & 134 & 249,2 & $6,25_{2}$ & - & - \\
\hline 86,8 & $\tilde{5}, 35_{6}$ & $5,30_{1}$ & 134 & 256,5 & $6,30_{3}$ & 一 & - \\
\hline 94,5 & $5,47_{5}$ & $5,41_{2}$ & 132 & 264,6 & $6,27_{8}$ & - & - \\
\hline 99,9 & $5,59_{3}$ & 5,52 & 124 & 272,7 & $6,2 t_{7}$ & - & - \\
\hline 105,3 & $5,64_{\tilde{\jmath}}$ & $5,57_{1}$ & 123 & & & $\cdot$ & \\
\hline
\end{tabular}

Tab. 7. Durchschnittliche Atomwärme von Rubidiumbromid.

an einem größeren Material mit der Erfahrung verglichen werden, während Blackman nur Werte für $\mathrm{NaCl}, \mathrm{KCl}$ und $\mathrm{KBr}$ zur Verfügung hatte. Wie aus Tab. 9 hervorgeht, passen die $\Theta_{\mathrm{e}}$-Werte recht gut mit den $\Theta_{\mathrm{D}}$-Werten zusammen, was allerdings lediglich einem Zufall zuzuschreiben ist. Denn die Übereinstimmung ist nach Blackman keineswegs von vornherein $\mathrm{zu}$ erwarten, da $\Theta_{\mathrm{e}}$ auf die Eigenschaften des Kristalls im langwelligen Bereich des elastischen Spektrums, $\Theta_{n}$ dagegen bei mäßig hohen Temperaturen (d.h. $T \sim \Theta_{\mathrm{D}}$ ) vorwiegend auf seinen kurzwelligen Anteil zurückgeht. Daß tatsächlich eiñe Kompensation verschiedener Einflüsse statthat, folgt schon aus der Vernachlässigung der Temperaturabhängigkeit von $r_{0}$ und $x$, für die wir einfach die Werte bei Zimmertemperatur benützt haben.

Für $\mathrm{AgCl}$ und $\mathrm{AgBr}$, die ebenfalls im Steinsalzgitter kristallisieren, fällt übrigens das Bild erheblich ungünstiger aus, wie folgende Zusammenstellung zeigt:

$\begin{array}{ccccc} & \varkappa \cdot 10^{6} \mathrm{~cm}^{2} / \mathrm{kg} & r_{0} \cdot 10^{8} \mathrm{~cm} & \Theta_{\mathrm{e}} & \Theta_{\mathrm{D}} \\ \mathrm{AgCl} & 2,4 & 2,77 & 272 & 1: 34 \text { bis } 213 \\ \mathrm{AgBr} & 2,7 & 2,88 & 200 & 120 \text { bis } 160\end{array}$

\begin{tabular}{|c|c|c|c|c|c|c|c|}
\hline$T^{0} \mathrm{abs}$ & $C_{p}$ & $C_{v}$ & $\Theta_{\mathrm{D}}$ & $T^{0}$ abs. & $C_{p}$ & $\bar{C}_{v}$ & $\Theta_{\mathrm{D}}$ \\
\hline $11,6_{8}$ & $0,64_{0}$ & 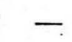 & 103 & 73,5 & $5,37_{0}$ & $5,30_{8}$ & 113 \\
\hline $12,0_{4}$ & $\mathbf{0 , 7 7 _ { 8 }}$ & & 99 & 78,7 & 5 & $5,37_{7}$ & 114 \\
\hline $12, b_{5}$ & $0,80_{5}$ & & 102 & 1,9 & $\overline{\mathbf{b}}, \mathbf{5} \mathbf{7}_{7}$ & $5,49_{4}$ & 118 \\
\hline $12,8_{4}$ & $0,84_{3}$ & 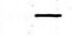 & 102 & 7,0 & $5,64_{5}$ & $5,55_{5}$ & 116 \\
\hline $12,9_{5}$ & $0,81_{9}$ & - & 104 & 102,2 & 5 & $0_{8}$ & 113 \\
\hline $13,5_{2}$ & $0,99_{1}$ & & 100 & 107,6 & 5,7 & $5,65_{2}$ & 111 \\
\hline $14,5_{7}$ & $1,0 \bar{\sigma}_{6}$ & & 105 & 112,7 & $5,81_{2}$ & $5,70_{2}$ & 106 \\
\hline $14,6_{9}$ & $1,09_{1}$ & & 105 & 117,9 & $5,86_{2}$ & $4_{4}$ & 101 \\
\hline $16,2_{9}$ & $1,27_{4}$ & $1,27_{3}$ & 109 & 123,3 & $5,88_{0}$ & $5,75_{6}$ & 102 \\
\hline $16,6_{4}$ & $1,35_{3}$ & $1,35_{2}$ & 108 & 129,0 & $5,92_{2}$ & $5,79_{0}$ & 97 \\
\hline $16,7_{5}$ & $1,33_{4}$ & $1,33_{3}$ & 109 & 134,4 & $5,93_{1}$ & $5,79_{4}$ & 100 \\
\hline $18,4_{5}$ & $1,56_{6}$ & 1,56 & 111 & 140,0 & 5,9 & $5,82_{1}$ & 95 \\
\hline $19,0_{5}$ & $1,64_{6}$ & $1,64_{4}$ & 112 & 144,9 & $5,97_{4}$ & $5,82_{5}$ & 97 \\
\hline $20,2_{6}$ & $1,82_{2}$ & $1,82_{0}$ & 113 & 3 & $6,00_{6}$ & $\mathbf{5}, 8$ & 92 \\
\hline 20,3 & $1,85_{9}$ & $1,85_{7}$ & 112 & 6 & $6,02_{8}$ & & 87 \\
\hline 21,4 & $1,99_{0}$ & $1,98_{8}$ & 113 & 2 & $6,04_{4}$ & 5 & 85 \\
\hline 22,3 & $2,13_{3}$ & $2,13_{0}$ & 113 &, 7 & $6,07_{6}$ & $5,89_{7}$ & 74 \\
\hline 22,8 & $2,17_{9}$ & $2,17_{6}$ & 115 & 172,2 & $6,08_{1}$ & $5,89_{6}$ & 78 \\
\hline 24,9 & $2,49_{3}$ & $2,48_{9}$ & 114 & 177,9 & $6,08_{5}$ & $\mathbf{5}, 89_{3}$ & 82 \\
\hline 24,8 & $2,51_{9}$ & $2,51_{4}$ & 114 & 184,6 & $6,08_{1}$ & $5,88_{3}$ & 92 \\
\hline $.27,3$ & $2,8 \bar{D}_{7}$ & 2,8 & 114 & 189,5 & $6,10_{5}$ & $5,90_{0}$ & 82 \\
\hline 29,8 & $3,15_{9}$ & 3,15 & 114 & 197,8 & $6,37_{5}$ & $6,14_{2}$ & - \\
\hline 32,3 & $3,43_{2}$ & $3,42_{1}$ & 114 & 203,4 & 6,1 & $5,95_{3}$ & - \\
\hline 3 & $3,67_{4}$ & $3,66_{1}$ & -115 & 208,8 & 6,1 & $5,92_{4}$ & 一 \\
\hline 3 & 3,9 & & 113 & 219,3 & $6,20_{1}$ & $5,95_{6}$ & - \\
\hline 39,8 & $4,09_{5}$ & $4,07_{6}$ & 114 & 225,1 & $6,19_{6}$ & $5,94_{5}$ & - \\
\hline 42,5 & $4,30_{1}$ & $4,27_{8}$ & 113 & 230,3 & $6,20_{6}$ & $\mathbf{5}, \mathbf{9 4}_{8}$ & - \\
\hline 45,6 & $4,48_{6}$ & $4,45_{9}$ & 113 & 235,7 & $6,21_{4}$ & $5,94_{9}$ & - \\
\hline 48,9 & $4,66_{7}$ & $4,63_{6}$ & 113 & 241,7 & $6,20_{2}$ & $5,93_{2}$ & - \\
\hline 3 & $4,81_{8}$ & 4,78 & 112 & 247,9 & $6,20_{3}$ & $5,92_{6}$ & - \\
\hline 56,6 & $4,90_{4}$ & $4,86_{5}$ & 116 & 254,3 & $6,21_{4}$ & $5,92_{9}$ & - \\
\hline 64 & $5,19_{2}$ & 5,14 & 112 & 260,5 & $6,23_{6}$ & $5,94_{1}$ & - \\
\hline 68,6 & $5,32_{3}$ & & 109 & 276,9 & $6,28_{4}$ & $5,97_{7}$ & - \\
\hline
\end{tabular}

Tab. 8. Durchschnittliche Atomwärme von Rubidiumjodid.

Die nach der Schmelzformel von Lindemann

$$
\Theta_{T_{\mathrm{e}}}=115 \sqrt{\frac{T_{\mathrm{e}}}{\widetilde{A} \widetilde{V}_{\mathrm{A}}^{2 / 3}}}
$$

aus der Schmelztemperatur $T_{\mathrm{e}}$, dem durchschnittlichen Atomgewicht $\widetilde{A}$ und dem durchschnittlichen Atomvolumen $\widetilde{V}_{\mathrm{A}}$ berechneten $\Theta_{T_{\mathrm{e}}}$-Werte beweisen (außer beim LiF!) aufs neue die Brauchbarkeit dieser alten Beziehung. Doch muß gesagt werden, daß der Zahlenfaktor angepaßt ist und von dem gleichfalls empirisch festgelegten Lindemannschen Wert 134 beträchtlich abweicht.

Recht gut stimmen die aus den Reststrahlen ermittelten $\Theta_{\mathrm{R}}$-Werte, wenn man die von Förster ling ${ }^{11}$ vorgeschlagene Korrektur nicht anbringt, durch die die Reststrahlfrequenz auf die Stelle maximaler Absorption umgerechnet wird. Benutzt man dagegen die ultraroten Absorptionsfrequen-

$$
{ }^{11} \text { K. Förster ling, Ann. Physik 61, } 577 \text { [1920]. }
$$




\begin{tabular}{|c|c|c|c|c|c|c|c|c|c|}
\hline \multirow{3}{*}{ Salz } & \multicolumn{9}{|c|}{ B erechnungsart } \\
\hline & \multicolumn{3}{|c|}{$\begin{array}{c}\text { Elastisch } \\
\text { nach Blackman }\end{array}$} & \multirow{2}{*}{$\begin{array}{c}\text { Nach } \\
\text { Linde- } \\
\text { mann } \\
\Theta_{T_{\mathrm{e}}}\end{array}$} & \multicolumn{2}{|c|}{$\begin{array}{c}\text { Aus den } \\
\text { Reststrahlen }\end{array}$} & \multicolumn{2}{|c|}{$\begin{array}{c}\text { Aus der } \\
\text { U-Rot- } \\
\text { Absorption }\end{array}$} & \multirow{2}{*}{$\begin{array}{c}\text { Aus der } \\
\text { spezif. Wärme } \\
\text { (diese Arbeit) } \\
\Theta_{D}\end{array}$} \\
\hline & $\begin{array}{l}\% \cdot 10^{6} \\
\mathrm{~cm}^{2} / \mathrm{kg}\end{array}$ & $r_{0} \cdot 10^{8} \mathrm{~cm}$ & $\Theta_{\mathrm{e}}$ & & in $\mu$ & $\Theta_{R}$ & in $\mu$ & $\Theta_{\mathbf{A}}$ & \\
\hline $\mathrm{LiF}$ & 1.50 & 2,01 & 686 & 1020 & 17 & 845 & 32,6 & 440 & $607-750$ \\
\hline $\mathrm{NaCl}$ & 4,18 & 2,81 & 292 & 294 & 52,0 & 276 & 61,1 & 235 & $275-300$ \\
\hline $\mathrm{KCl}$ & 5,53 & 3,14 & 233 & 229 & $63,{ }_{1}$ & 226 & 70,7 & 203 & 218-235 \\
\hline $\mathrm{KBr}$ & 6,56 & 3,29 & 185 & 171 & 81, & 176 & 88,3 & 162 & $152-183$ \\
\hline KJ & 8,37 & 3,52 & 162 & 119 & 94,0 & 153 & 102 & 141 & $115-200$ \\
\hline $\mathrm{RbBr}$ & 8,0 & 3,43 & 136 & 123 & $\sim 100$ & 143 & 114 & 126 & $120-135$ \\
\hline $\mathrm{RbJ}$ & 9,1 & 3,67 & 119 & 109 & $\sim 117$ & 122 & 129, & 111 & $100-118$ \\
\hline
\end{tabular}

Tab. 9. Vergleich verschiedener Berechnungsarten der charakteristischen Temperatur der Alkalihalogenide.

zen von $\mathrm{Czerny}$ und $\mathrm{Barnes}{ }^{12}$, so ist die Übereinstimmung für die Salze mit langwelligen Reststrahlen zwar noch ausreichend (RbJ, RbBr, $\mathrm{KJ}, \mathrm{KBr}$ ), sie geht aber schon beim $\mathrm{KCl}$ und $\mathrm{NaGl}$ und besonders $\mathrm{kra} ß$ beim $\mathrm{LiF}$ verloren.

4. Wenden wir uns nun an Hand von Abb. 1 den experimentellen $\Theta_{\mathrm{D}}$-Werten zu. Zunächst fällt das fächerförmige Auseinanderlaufen der $\Theta_{\mathrm{D}}$-Werte nach der Ordinate hin auf. Hinter dieser, durch das jetzt vorliegende Beobachtungsmaterial aufgedeckten Tatsache scheint sich eine Gesetzmäßig- keit zu verbergen, die durch die Größe der Atommassen und die Absolutwerte der charakteristischen Temperaturen beherrscht wird. Weiter bemerken wir das Versagen der Kontinuumstheorie bei $\mathrm{LiF}, \mathrm{NaCl}$ und $\mathrm{KCl}$ im Gebiet des $T^{3}$-Gesetzes, das in Abb. 1 schraffiert ist und bei $\Theta_{D} / T$-Werten $>12$ erreicht werden sollte. Auf diese Verhältnisse sind wir beim Lithiumfluorid bereits näher eingegangen, so daß wir hier nur darauf zu verweisen brauchen ${ }^{13}$. Je höher die charakteristische Temperatur, um so ausgeprägter ist der Anstieg der $\Theta_{D}$-Werte nach tiefen Temperaturen hin, gleichzeitig rückt das flache Minimum bei LiF, $\mathrm{NaCl}$ und $\mathrm{KCl}$ nach tieferen Temperaturen. Bei $\mathrm{KBr}, \mathrm{KJ}, \mathrm{RbB}$ und RbJ ist schließlich nur der abfallende Ast übrig, und es muß z. Zt. offen bleiben, ob hier bei Heliumtemperaturen überhaupt ein Minimum erreicht wird. Alle Salze zeigen überdies mehr oder weniger ausgeprägte Maxima.

5. Das Versagen der Debyeschen Theorie bei Ionenkristallen führt dazu, daß man den Einfluß der Gitterstruktur doch berücksichtigen muß, indem man das Spektrum des Festkörpers berechnet

12 R. B. Barnes u. M. Czerny, Z. Physik 72, 447 [1931]; R. B. B a r n e s, Z. Physik 75, 72:3 [1932]. 13 K. Clusi us, Z. Naturforschg. 1, 71 [1946].

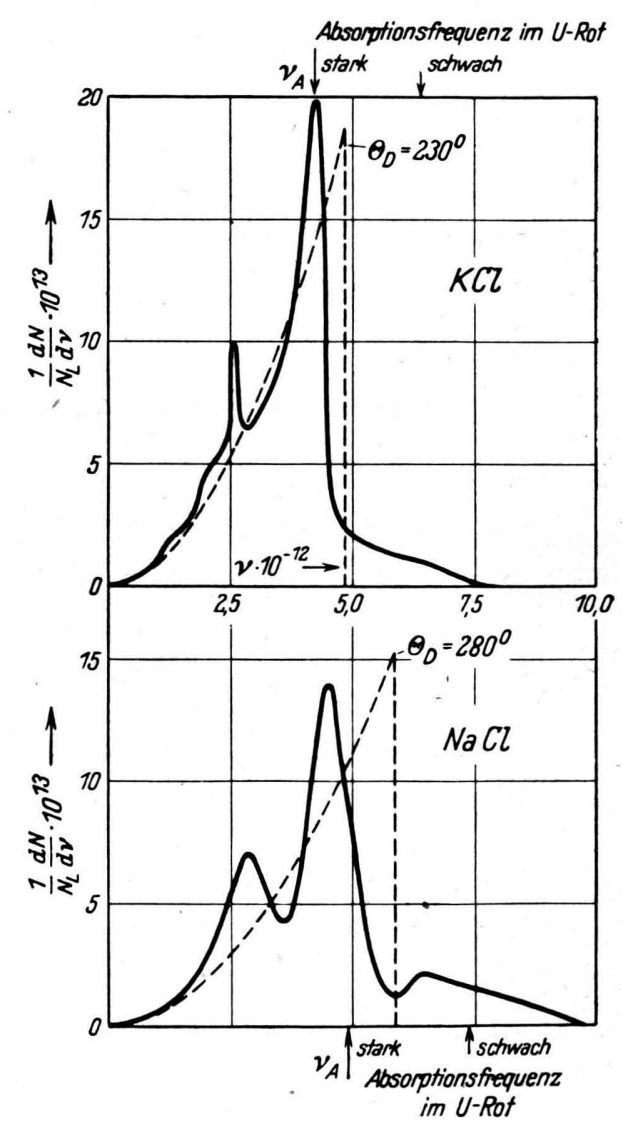

Abb. 2. Spektren des festen $\mathrm{KCl}$ und $\mathrm{NaCl}$ nach der Gittertheorie (ausgezogene Kurven) und idealisierte Debyesche Spektren (gestrichelte Kurven).

und aus ihm die Molwärmen ableitet. B or $n$ und v. $\mathrm{Karman}{ }^{14}$ fanden bei einer linearen Ionenkette, daß das Spektrum im allgemeinen in einen kurzwelligeren optischen und einen langwellige-

${ }_{14}$ M. B orn u. v. K a rma n, Physik. Z. 13, 297 [191:]. 
ren akustischen Zweig aufspaltet. Blackman ${ }^{15}$ wandte Borns Verfahren auf das zweidimensionale Gitter an und war bereits in der Lage, über das allgemeine Verhalten der $\Theta_{D}$-Werte von Ionenkristallen zutreffende Angaben zu machen. Die höchst umfangreichen Rechnungen für räumliche Kristallgitter hat $\mathrm{Ke}$ ll e r m a n $\mathrm{n}^{16}$ für $\mathrm{NaCl}$ und $\mathrm{J}_{0} \mathrm{na}^{17}$ für $\mathrm{KCl}$ durchgeführt.

Das wahre Spektrum des Kristalls unterscheidet sich von der Debyeschen Idealisierung ganz erheblich, wie Abb. 2 zeigt. Kellermann fand beim Natriumchlorid drei Häufungsstellen der Frequenz, von denen die intensivste nahe an der ultraroten Absorptionsbande liegt. Jo $\mathrm{na}^{17}$ hat beim Kaliumchlorid nur zwei Maxima über einem breiteren Untergrund erhalten, die Abweichung vom Debyeschen Spektrum ist hier nicht ganz so stark. Das Hauptmaximum fällt mit der ultraroten Absorptionsfrequenz zusammen. Die Spektren der andern Salze sind noch unbekannt ${ }^{18}$.

6. Wir führen nunmehr den Vergleich von Experiment und Theorie für die spezifischen Wärmen von $\mathrm{NaCl}$ und $\mathrm{KCl}$ durch. Auf Abb. 3 sind die für Natriumchlorid gefundenen $\Theta_{D}$-Werte eingezeichnet und durch eine ausgezogene Kurve miteinander verbunden. Der von Kellermann theoretisch aus dem wahren Spektrum berechnete Verlauf ist gestrichelt eingetragen. Die Übereinstimmung ist vorzüglich, da nicht nur der allgemeine Kurvencharakter, wie das Minimum des Pseudo- $T^{3}$-Gebiets, sondern auch der Absolutwert richtig ist. Die größte, bei $60^{\circ}$ abs. auftretende Abweichung der $\Theta_{D}$-Werte entspricht nur einer Differenz von $2 \%$ in der Atomwärme, einem experimentellen Wert von

15 M. B l a c k m a n, Proc. Roy. Soc. [London], Ser. A 148, 365, 384 [1935]; 149, 117, 126 [1935].

16 E. W. K e 1 l e r m a n n, Philos. Trans. Roy. Soc. London, Ser. A 238, 513 [1940]; Proc. Roy. Soc. [London], Ser. A 178, 17 [1941].

17 M. J o n a, Physic. Rev. 60, 822 [1941].

18 Die optischen Eigenschaften des LiF sind von der Pohlschen Schule festgestellt worden: K. K o r th, Nachr. Ges. Wiss. Göttingen, math.-physik. Kl. 1, 187 [1935]]; H. W. Ho h ls, Ann. Physik 29, 433 [1937].
$C_{v}=2,50 \mathrm{cal} /{ }^{\circ}$ steht der theoretische von $2,45 \mathrm{cal} /{ }^{\circ}$ gegenüber.

Schließlich sind auf Abb. 4 die beobachteten und berechneten Werte für Kaliumchlorid vereinigt. Die Heliummessungen von $\mathrm{K}$ e e s o $\mathrm{m}$ und $\mathrm{Clark} \mathrm{k}^{12}$ schließen sich unseren Daten gut an, sie liegen
Abb. 3. Experimenteller Verlauf der $\Theta_{D}$-Werte beim $\mathrm{NaCl}$ zogene Kurve) und aus dem unteren Spektrum von Abb. 2 berechneter Verlauf (gestrichelte Kurve).

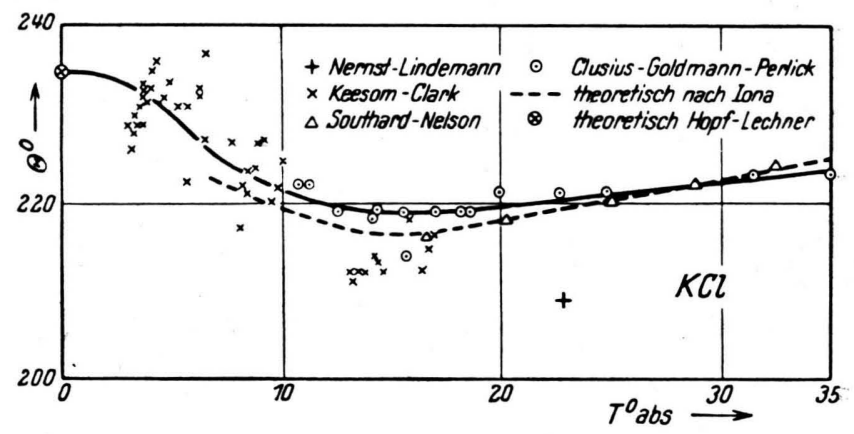

Abb. 4. Experimenteller Verlauf der $\Theta_{D}$-Werte beim $\mathrm{KCl}$ (ausgezogene Kurve) und aus dem oberen Spektrum von Abb. 2 berechneter Verlauf (gestrichelte Kurve).

jedoch im Gebiet des flüssigen Wasserstoffs etwas tiefer. Geringer ist die Abweichung der von Southard und Nelson ${ }^{20}$ oberhalb von $15^{\circ}$ abs. veröffentlichten Werte. Der von $\mathrm{H}$ opf und Lech ner ${ }^{21}$ aus der Schallgeschwindigkeit berechnete $\Theta_{\mathrm{D}}$-Wert von $235^{\circ}$ für den abs. Nullpunkt liegt

19 W. H. K e e s o m u. C. W. C l a r k, Physica 2 , 698 [1935]; Commun. Kamerlingh Omnes Lab. Univ. Leiden 2380.

20 J. C. Southard u. R. A. Nelson, J. Amer. chem. Soc. 55, 4865 [1933].

21 J. H op f u. G. L e c hn e r, Verh. dtsch. physik.. Ges. 16, 643 [1914]. 
auf der Verlängerung der experimentellen Kurve. Jona berechnete aus dem Spektrum Abb. 2 einen Kurvenverlauf, der den Habitus der Beobachtungen ausreichend wiedergibt. Besonders wird das bei $15^{\circ}$ abs. von Jona geforderte Minimum durch unsere Messungen durchaus bestätigt.

Insgesamt kommen wir also zu dem Ergebnis, daß die Bornsche Gittertheorie in der von Blackman, Kellermann u. a. weitergeführten Form den tatsächlichen Verhältnissen bei den Alkalihalogeniden $\mathrm{NaCl}$ und $\mathrm{KCl}$ ausgezeichnet gerecht wird. Eine Ausdehnung der Rechnungen auf andere Beispiele, besonders auf $\mathrm{LiF}$, ist höchst wünschenswert.
7. Man darf nicht übersehen, daß der praktische Wert des $T^{3}$-Gesetzes bei den Alkalihalogeniden glücklicherweise keine Einschränkung erfährt. Wahrscheinlich kann dieser Befund dahin verallgemeinert werden, daß es zur Berechnung des Energieinhalts und der Entropie immer statthaft sein wird, die Debyesche Funktion mit der bei $10^{\circ}$ abs. gefundenen charakteristischen Temperatur zur Extrapolation bis zum absoluten Nullpunkt zu benutzen. Dies ist nicht nur die bequemste, sondern bislang die beste Näherung, über die wir verfügen.

Zur Veranschaulichung ermitteln wir für $\mathrm{NaCl}$ 1. mit $\Theta_{\mathrm{D}}=301,5^{\circ}$ nach dem $T^{3}$-Gesetz den Energieinhalt $U$ und die Entropie $S$ rechnerisch, und 2. nach dem auf Abb. 3 gezeigten Verlauf der $\Theta_{D}$-Werte graphisch von 0 bis $10^{\circ}$ abs.:

$$
U=\int_{0}^{10} C_{v} d T
$$

Rechnerisch mit $\Theta_{\mathrm{D}}=301,5^{\circ}$

Graphisch mit $\Theta_{\mathrm{D}}=f(T)$
Der Unterschied ist bei der gegenwärtigen Meßgenauigkeit belanglos, da die experimentell bestimmte Entropie bei Zimmertemperatur durch die Unsicherheit fast aller Molwärmen bei höheren Temperaturen ohnehin mit einem mittleren Fehler von $\pm 0,05$ Clausius behaftet ist.

8. Man muß sich fragen, ob die von F. Simon und Mitarbb. bei komplizierter zusammengesetzten Verbindungen empirisch festgestellte Gültigkeit des $T^{3}$-Gesetzes nur vorgetäuscht ist oder nicht. Das Versuchsmaterial läßt in vielen Fällen durchaus die Möglichkeit zu, daß stets nur ein Pseudo- $T^{3}$-Gebiet erreicht wurde. Unseres Er- achtens bleibt die Frage also offen. Es ist durchaus denkbar, daß das eigentliche $T^{3}$-Gebiet mancher Substanzen viel weniger ausgedehnt ist und erst bei viel tieferen Temperaturen erreicht wird, als man bisher angenommen hat. Weitere Versuche an einem möglichst umfassenden Material bei möglichst tiefen Temperaturen sind daher angezeigt.

Die vorliegenden Messungen wurden bereits in den Jahren 1933/34 im Physikalisch-Chemischen Institut der Universität Göttingen ausgeführt. Der eine von uns (J. Goldmann) dankt der Deutschen Forschungsgemeinschaft für ein zweijähriges Stipendium. 